\title{
Maternal mortalities due to infectious diseases at a tertiary care centre in India
}

\author{
Meena Naresh Satia*, Shruti Ashok Panchbudhe, Manali Prakash Shilotri
}

\begin{abstract}
Department of Obstetrics and Gynaecology, Seth Gordhandas Sunderdas Medical College and King Edward VII
\end{abstract} Memorial Hospital, Mumbai, Maharashtra, India

Received: 26 May 2016

Accepted: 15 June 2016

\section{*Correspondence:}

Dr. Meena Naresh Satia,

E-mail: meenasatia@kem.edu

Copyright: (c) the author(s), publisher and licensee Medip Academy. This is an open-access article distributed under the terms of the Creative Commons Attribution Non-Commercial License, which permits unrestricted non-commercial use, distribution, and reproduction in any medium, provided the original work is properly cited.

\begin{abstract}
Background: Maternal death and poor birth outcomes are major public health issues in much of the world. Most of the mortality and morbidity burden around child birth are directly due to obstetrical problems such as haemorrhage. However, there is very little information on the importance of the great diversity of infectious diseases on pregnancy outcome, especially in the tropics. There is poor evidence as to whether obstetrics patients are at a higher risk of diseases that are common causes of fever in the tropical areas and what the impact is on mothers and their offspring, by pathogens and gestational age. Understanding of this fact is impaired as deaths from infectious diseases and deaths in pregnancy are often not evaluated jointly in public health surveillance.

Methods: This is a retrospective, observational case series undertaken at a tertiary care centre at Seth G. S. Medical College and K.E.M. Hospital, Mumbai, Maharashtra, India during the period of January 2011 to December 2015.This data collection was done by reviewing the maternal death record forms.

Results: This study shows that there is significant number $(23.58 \%)$ of maternal deaths due to infectious diseases. Out of a total of 37266 deliveries over a period of five years, 407 maternal deaths occurred and out of these 96 patients died due to infectious diseases. Among these, deaths due to tuberculosis were seen in $32.29 \%$, hepatitis $\mathrm{E}$ $26.04 \%$, pyrexia of unknown origin $9.3 \%$, malaria $8.33 \%$, dengue $6.25 \%$, swine flu $5.2 \%$, leptospirosis $3.12 \%$, and community acquired pneumonia $3.12 \%$, viral encephalitis $2.08 \%$, tuberculosis with HIV $2.08 \%$, amoebiasis and typhoid $1.04 \%$ each.

Conclusions: Though India failed to achieve the millennium development goal, it fell short of the goal by a small margin. Educational status and the socioeconomic development are major factors that need to be corrected. Effective preventive strategies at personal and community level will definitely reduce the preventable maternal mortalities due to infectious diseases and aid India in achieving further targets.
\end{abstract}

Keywords: Maternal mortality, Infectious diseases

\section{INTRODUCTION}

The study of causes of maternal mortality and formulation of measures to prevent it has been an ongoing quest. The leading causes of maternal deaths vary between different regions of the world, primarily being influenced by the socio-economic status and level of education of the people in those parts. Developed countries mainly face maternal deaths due to indirect causes, direct obstetric causes and hypertensive disorders while the developing regions have haemorrhage and indirect aetiologies as the major causes. ${ }^{1}$ In developing countries like India, there are several infectious diseases such as tuberculosis, hepatitis E, swine flu and vector borne diseases like malaria, dengue, leptospirosis that are endemic and many occurring in epidemics. Break-outs of these diseases are influenced by weather changes and climatic events in these countries, especially those in the 
tropical regions. In India, the monsoons are associated with a higher incidence of infectious diseases. They are responsible for adversely affecting the general population of these regions and also pose a substantial threat to maternal health. It is presupposed that the physiological changes of pregnancy like suppression of the T-cell mediated immunity and high levels of hormones like steroids, oestrogen and progesterone contribute to development of severe forms of infectious diseases and lead to maternal mortality.,3 As the incidence of infectious diseases is high in developing countries like India, with lower respiratory tract infections, HIV/AIDS, diarrhoeal diseases, malaria, tuberculosis etc. being the leading causes of death in low-income countries, it is imperative to determine the burden of these diseases on maternal mortality. ${ }^{4}$

As per the millennium development goals (MDG), India aimed at reducing maternal mortality ratio (MMR) by $75 \%$ from 1990 till 2015. The MMR of India has reduced from 560/lakh live births in 1990 to $167 /$ lakh live births in 2011-2013 i.e. a 70.17\% decline. ${ }^{5}$ There has always been a focus on development of measures to reduce direct obstetric deaths as many of them are preventable. As a decreasing trend is seen in the deaths due to direct obstetric causes, there should now be a shift of focus to other causes of maternal mortalities. Infectious diseases causing maternal deaths are also preventable to a large extent. A thorough evaluation of the extent to which infectious diseases contribute to maternal mortality and the proportion of indirect obstetric deaths caused by infectious diseases will help formulate health policies. This could have aided our country in achieving the MDGs by 2015. Nevertheless, it can now help us to explore different measures to further reduce our maternal mortality rate. Thus, our study will determine the incidence of infectious diseases leading to maternal mortalities at a tertiary care centre in India.

\section{METHODS}

The study was initiated after seeking approval from Institutional ethics committee. This is a hospital-based retrospective study which was conducted at a tertiary hospital in the year 2011 to 2015 . We recruited all cases of maternal mortality due to infectious diseases during this period.

The method of collection of data was the case record forms of these maternal mortality patients were reviewed with respect to infectious diseases. We focused on specific diseases that local evidence suggested might be important, including malaria, leptospirosis, dengue fever, swine flu, typhoid and hepatitis E infection etc.

\section{Inclusion criteria}

Deaths resulting from infectious diseases during pregnancy itself, labour, during puerperium or within 42 days of termination of pregnancy, irrespective of site and duration of pregnancy were included.

\section{Exclusion criteria}

Deaths from other direct or indirect causes leading to maternal mortality during this period were excluded.

\section{RESULTS}

Table 1: Year-wise maternal death distribution with total MMR-1125.6/ lakh live births.

\begin{tabular}{|lllllll|}
\hline Year & 2011 & 2012 & 2013 & 2014 & 2015 & Total \\
\hline Total confinements & 7036 & 8053 & 8621 & 6776 & 6780 & 37266 \\
\hline Total live births & 6693 & 7873 & 8435 & 6598 & 6557 & 36156 \\
\hline Total maternal deaths & 83 & 80 & 97 & 70 & 77 & 407 \\
\hline
\end{tabular}

Table 2: Distribution of maternal deaths by causes.

\begin{tabular}{|lllllll|}
\hline Year & 2011 & 2012 & 2013 & 2014 & 2015 & Total \\
\hline Total maternal deaths & 83 & 80 & 97 & 70 & 77 & 407 \\
\hline Maternal deaths due to Infectious diseases & 24 & 16 & 21 & 23 & 12 & 96 \\
\hline Maternal deaths-direct obstetric causes & 13 & 21 & 22 & 15 & 19 & 90 \\
\hline Maternal deaths-indirect causes (excluding infections) & 46 & 43 & 54 & 32 & 46 & 221 \\
\hline
\end{tabular}

This study shows that there is a significant number $(23.58 \%)$ of maternal deaths due to infectious diseases. Out of a total of 37,266 deliveries over a period of five years, 407 maternal deaths occurred and out of these, 96 patients died due to infectious diseases. Among these deaths due to tuberculosis was seen in $32.29 \%$, hepatitis E $26.04 \%$, pyrexia of unknown origin $9.3 \%$, malaria $8.33 \%$, dengue $6.25 \%$, swine flu $5.2 \%$, leptospirosis $3.12 \%$, community acquired pneumonia $3.12 \%$, viral encephalitis $2.08 \%$, tuberculosis with HIV $2.08 \%$, amoebiasis and typhoid $1.04 \%$ each. 
Table 3: Causes of maternal deaths due to infectious diseases.

\begin{tabular}{|lllllll|}
\hline Year & 2011 & 2012 & 2013 & 2014 & 2015 & Total (\%) \\
\hline Febrile Illness ( pyrexia of unknown origin) & 2 & 0 & 4 & 3 & 0 & $9(9.3)$ \\
\hline Malaria & 2 & 3 & 2 & 0 & 1 & $8(8.33)$ \\
\hline Leptospirosis & 1 & 0 & 1 & 0 & 1 & $3(3.12)$ \\
\hline Dengue & 1 & 1 & 3 & 1 & 0 & $6(6.25)$ \\
\hline Hepatitis E & 12 & 7 & 0 & 4 & 2 & $25(26.04)$ \\
\hline Tuberculosis & 6 & 4 & 9 & 9 & 3 & $31(32.29)$ \\
\hline Tuberculosis with HIV & 0 & 0 & 1 & 0 & 1 & $2(2.08)$ \\
\hline Swine flu & 0 & 0 & 0 & 1 & 4 & $5(5.2)$ \\
\hline Typhoid & 0 & 0 & 1 & 0 & 0 & $1(1.04)$ \\
\hline Amoebiasis & 0 & 1 & 0 & 0 & 0 & $1(1.04)$ \\
\hline Community acquired pneumonia & 0 & 0 & 0 & 3 & 0 & $3(3.12)$ \\
\hline Viral encephalitis & 0 & 0 & 0 & 2 & 0 & $2(2.08)$ \\
\hline Total deaths due to infectious causes & 24 & 16 & 21 & 23 & 12 & 96 \\
\hline
\end{tabular}

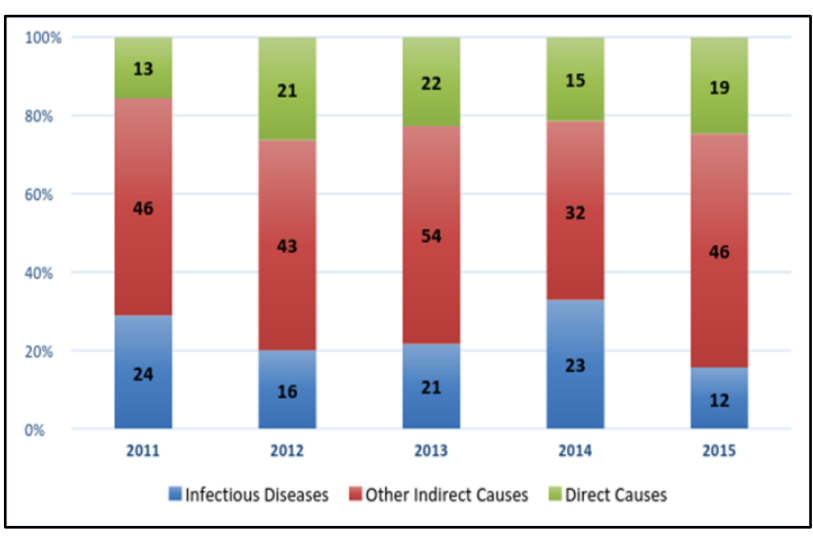

Figure 1: Percentage distribution of maternal deaths by causes.

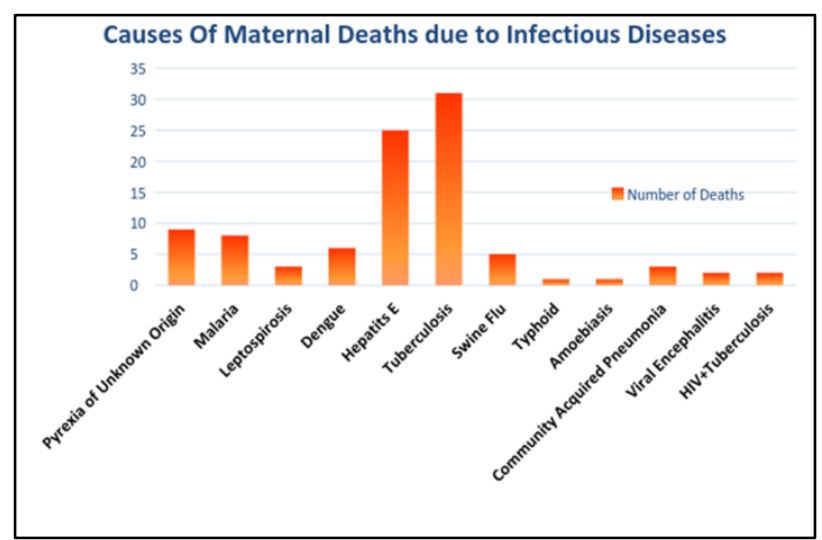

Figure 3: Bar diagram of various causes of infectious diseases leading to maternal mortality.

DISCUSSION

Table 4: Comparative studies.

\begin{tabular}{|c|c|c|c|c|c|c|}
\hline Study (author) & $\begin{array}{l}\text { Total } \\
\text { maternal } \\
\text { deaths }\end{array}$ & $\begin{array}{l}\text { Indirect } \\
\text { obstetric } \\
\text { deaths }\end{array}$ & $\begin{array}{l}\text { Deaths due to } \\
\text { infectious } \\
\text { diseases }\end{array}$ & $\begin{array}{l}\text { Deaths due to } \\
\text { tuberculosis }\end{array}$ & $\begin{array}{l}\text { Deaths due to } \\
\text { malaria }\end{array}$ & $\begin{array}{l}\text { Deaths due to other } \\
\text { infectious diseases }\end{array}$ \\
\hline $\begin{array}{l}\text { Ahmed } Y \text { et al } \\
\text { conducted at } \\
\text { Zambia }^{6}\end{array}$ & 251 & $145(58 \%)$ & $77 \%$ & $25 \%$ & $30 \%$ & $22 \%$ \\
\hline Our study & 407 & $221(54.29 \%)$ & $23.58 \%$ & $7.6 \%$ & $1.9 \%$ & $14 \%$ \\
\hline
\end{tabular}

Every 10 minutes a woman dies in India from pregnancy and complications during labour. Maternal mortality is the direct reflection of the state of maternal health and the quality of service we cater to people. Maternal health refers to the health of women during pregnancy, childbirth and the postpartum period and it encompasses the health care dimensions of family planning, preconception, prenatal, and postnatal care. Although direct obstetric causes are the major burden in developing countries, indirect causes due to infections are increasing and special emphasis should be focused on these causes. The various infectious diseases which were found important in our study are discussed and also the comparative statistics with other studies is shown in the table below. The table shows that indirect obstetric causes of maternal mortality due to infectious diseases 
are on a rise and tuberculosis is the leading infection causing maternal mortality in developing countries.

\section{Hepatitis $\boldsymbol{E}$}

Viral hepatitis especially hepatitis E is associated with increased risk of mortality accounting for $0.8-29.4 \%{ }^{7}$ hepatitis $\mathrm{E}$ is usually self-limiting but may develop into fulminant hepatitis (acute liver failure). The hepatitis $\mathrm{E}$ virus is transmitted because of contamination of food and water due to poor hygienic conditions. Hepatitis $\mathrm{E}$ is found worldwide, but the prevalence is highest in South East Asia due to poor sanitary and hygienic conditions, overcrowding, illiteracy and poverty which perpetuate the spread of this infection. Water supply contaminations with human faeces is a frequent source of epidemics and mainly affect young adults, the clinical attack rate being highest among those aged 15-35 years. Reported causes of death in pregnancy include hepatic encephalopathy with fulminant hepatic failure and disseminated intravascular coagulation. Management should be predominantly preventive, relying on clean drinking water, good sanitation, and proper personal hygiene. Care should be taken while consuming uncooked fruits/vegetables. Boiling water may prevent infection, but the effectiveness of chlorination is unknown.

\section{Malaria}

Malaria in pregnancy with any of the two parasites Plasmodium vivax or plasmodium falciparum, or both are potentially dangerous and is known to cause higher rates of miscarriage, IUGR/IUFD, premature delivery, and neonatal death. Over 50 million of pregnant women are exposed to the risk of malaria in pregnancy and it has been reported that malaria infection is directly responsible for 75,000-20,00,000 perinatal deaths every year. ${ }^{8}$ Pregnant women with malaria are 3 times more likely to suffer from severe disease compared with their non-pregnant counterparts, The mortality rate from severe disease is $50 \%$ and in areas which are endemic for malaria, it is estimated that at least one fourth of pregnant women are infected with malaria, with the highest risk for infection and morbidity in adolescents, primigravida and those co-infected with $\mathrm{HIV}^{8-10}$ The current WHO recommendations for prevention of malarial disease in pregnancy relies on providing women with insecticidetreated bed nets and intermittent presumptive treatment. ${ }^{11}$

\section{Dengue}

Dengue fever during pregnancy usually causes no serious harm. Dengue infection is a febrile illness caused by dengue virus serotypes (designated DENV-1, DENV-2, DENV-3, and DENV-4) of the genus Flavivirus, family Flaviviridae and is the most important mosquito-borne infection caused by Aedes Aegyptus. It usually causes no serious harm during pregnancy. The clinical severity of disease has a wide spectrum and according to the WHO dengue classification scheme, there are four grades ranging from uncomplicated dengue fever (DF) to dengue hemorrhagic fever (DHF) and devastating dengue shock syndrome (DSS). Infection by one serotype produces lifelong immunity to that specific serotype but only a few months of immunity to the others. Dengue infection in pregnancy carries the risk of haemorrhage for both the mother and the new-born. There is also a risk of premature birth and foetal death and vertical transmission causing neonatal thrombocytopenia that necessitates platelet transfusions. ${ }^{12-14}$ Diagnosis of dengue infection affects decisions about the mode of delivery due to the potential risk of haemorrhage secondary to thrombocytopenia. Elevated liver enzymes, haemolysis and low platelet counts may be confused with the diagnosis of haemolysis, elevated liver enzymes, low platelet count (HELLP) syndrome, which occurs in women with pre-eclampsia and eclampsia. Positive serology/viral PCR studies confirm dengue infections. Dengue in pregnancy is associated requires early diagnosis and conservative treatment which includes proper hydration, antipyretics and careful monitoring. Health-care providers should consider dengue in the differential diagnosis of pregnant women with fever during epidemics in endemic areas, and be cautious that clinical presentation may be atypical and early diagnosis is made difficult by the ambiguity of clinical findings and physiological changes of pregnancy that may confuse the clinician. Platelet count may fall rapidly but no active intervention required unless patient is in labour or has bleeding disorder. Gestational age of occurrence of dengue fever seems to have a role early or late in pregnancy had a poor prognosis. Severe thrombocytopenia requiring prompts resuscitation with blood and blood products prior to and during delivery, and was a key point in successful outcome of individuals having platelet count. Dengue prevention and control is implemented through the bi-regional dengue strategy (2008-2015) of the WHO South-East Asia and Western Pacific regions which consists of six elements: dengue surveillance, case management, outbreak response, integrated vector management, social mobilization and communication for dengue and dengue research (a combination of both formative and operational research). ${ }^{15}$

\section{HIVIAIDS}

Women aged 15-44, HIV/AIDS is the leading cause of death globally. Recent data suggest that pregnant or women in postnatal period living with HIV have approximately eight times higher maternal mortality than those who are not infected. ${ }^{16}$ The confidential enquiries made between 2008 and 2010 in South Africa suggest that most deaths in $\mathrm{HIV}$-infected pregnant and postpartum women are due to infections including pneumonia, tuberculosis, and meningitis. ${ }^{17}$ HIV also appears to increase the risk of infections in pregnancy, childbirth, and postpartum, with HIV-infected women experiencing three times the risk of puerperal sepsis. ${ }^{18}$ WHO guidelines of 2013 state that all pregnant and 
breast-feeding women should be initiated on antiretroviral therapy, irrespective of their CD4 counts. This will contribute to reducing HIV-related maternal mortality.

\section{Tuberculosis}

The highest rates of TB are found in sub-Saharan Africa, India, China, and the islands of Southeast Asia and is responsible for 6 to $15 \%$ of maternal mortality. ${ }^{19} \mathrm{~TB}$ infection also has great impact on the health of the newborns; pregnant women with pulmonary TB have approximately twice the risk of delivering a baby who is premature or low birth weight and six times the risk of a perinatal death. TB and TB-HIV co-infection are usually associated with IUGR/IUFD, prematurity and deteriorating maternal health. Pregnant women living with HIV have 10 times the risk of developing active TB, and pregnant women with HIV/TB co-infections face higher risks of maternal mortality. HIV-infected women with active TB are also more than two times as likely to transmit HIV to their infants. ${ }^{20,21}$

\section{Leptospirosis}

It is a rare and severe infection with leptospira bacteria found in fresh water that has been contaminated by animal urine. Leptospirosis occurs worldwide but is most common in tropical regions with high rainfall. The various risk factors for infection include recreational activities includes various water-based activities, occupational exposure and household exposure to domesticated animals, infestation of rodents and contaminated rainwater catchment systems. Infected rodents are known to shed the organism via urine throughout life, leading to contamination of the environment. The disease occurs when humans come into contact with the urine polluted environment. Rare cases have been reported when it is spread through breast milk or from a mother to her unborn child through placenta. The outcomes of leptospirosis during pregnancy are varied which includes foetal loss and miscarriage, stillbirth, congenital infection and oligohydramnios as well as good outcomes with healthy neonates. ${ }^{22}$ Treatment with effective antibiotics should be initiated as soon as the diagnosis of leptospirosis is suspected and clinicians should never wait for the results of laboratory tests before starting treatment with antibiotics. This is because serological tests do not become positive until about a week after the onset of illness, and cultures may not become positive for several weeks. The preventive measures include avoidance of areas of stagnant water, especially in tropical climates and rodent control.

\section{Swine flu}

Human infection with the novel H1N1 strain of the influenza A virus which was formerly called swine flu was first identified in April 2009. ${ }^{23}$ There have been outbreak which have reached pandemic status. Pregnant women are especially susceptible for the development of complications and have a 4 to 5 fold increased rate of serious illness and hospitalization with influenza. It is crucial that all obstetric care providers be familiar with the symptoms, treatment, and prevention of $\mathrm{H} 1 \mathrm{~N} 1$ infection in pregnant women. Patients usually present with symptoms of an acute respiratory illness which includes cough, sore throat, rhinorrhea, and fever and others with nonspecific complaints like headache, fatigue, body aches, vomiting, and diarrhoea. Also clinical presentation sometimes can be complicated by development of a secondary bacterial infection such as pneumonia. Pregnancy-related complications H1N1infection includes febrile morbidity and nonreassuring foetal testing which includes foetal tachycardia. Hyperthermia in early pregnancy causes neural tube defects and other congenital anomalies, and fever during labour and childbirth is a risk factor for neonatal seizures, new born encephalopathy, cerebral palsy, and death. ${ }^{24}$ The best way to reduce the risk of spreading the virus is to observe good respiratory hygiene by using face mask and disposing of it immediately. Pregnant women admitted with respiratory complications should be managed jointly by a multidisciplinary team between the obstetrician and medical experts. Woman in labour should be best managed in the delivery Unit with assistance from respiratory team and the obstetric anesthetic team. Treatment with antivirals should be started on clinical grounds whilst awaiting test results. The two types of antiviral drugs known to be effective against the swine flu virus are oseltamivir (Tamiflu®) and zanamivir (Relenza $\left.{ }^{\circledR}\right)$ which are neuraminidase inhibitors and act by stopping the virus from budding and escaping from the host cells. Oseltamivir and zanamivir are both pregnancy category $\mathrm{C}$ drugs, but no adverse events have been reported to date among women who received these agents during pregnancy. ${ }^{25}$ Oseltamivir is given in the form of oral capsules and zanamivir is given as an inhaler. The safety of these antiviral drugs in pregnancy has been looked at from various experiences and it has been found that oseltamivir although crosses the placenta and breast milk in small amounts, no adverse effects on the foetus or pregnancy have been recorded. Zanamivir is the drug of choice due to its limited systemic absorption, an inhaled route of administration may not be tolerated especially in women those who have underlying respiratory disease such as asthma or chronic obstructive pulmonary disease in which conditions oseltamivir is a reasonable alternative. Treatment should ideally be started within 48 hours of the symptoms due to its greatest benefit during this period and should be continued for 10 days after the last known exposure, but may need to be extended in clinical settings where multiple exposures are likely to occur. Studies have shown that swine flu vaccination during pregnancy can pass antibodies on to the baby that will protect against flu for six months after birth. CDC recommends that pregnant women get them vaccinated during any trimester of their pregnancy to protect themselves, unborn babies, and their newborns from flu. The common side 
effects experienced by pregnant women are mostly the same as experienced by general people and includes soreness, redness, and/or swelling at the site of injection, fainting, headache, fever, muscle aches, nausea and fatigue. Side effects occur after the injection is given and usually last for 1-2 days. Those with severe lifethreatening allergy to any of the vaccine ingredients should not be given the injection. Breastfeeding is not a contraindication to flu vaccine and it reduces mothers' risk of getting flu and also reduces the risk to their babies which is important for children younger than 6 months old since they are too young to be vaccinated.

\section{CONCLUSION}

This study shows that there is significant number of indirect causes of maternal mortality is attributed to infectious diseases (25.3\%). These infectious diseases can occur at any time during pregnancy or postpartum period and their severity will vary depending on the virulence of the agent, the susceptibility and gestational age of the foetus, and the route of the infection. The likelihood for transmission from mother to the foetus depends upon the time and type of maternal infection and maternal immune responses, whereas the severity of foetal damage once infection has established is strictly related to interaction among the foetal immune response and the pathogenicity of the infectious agent. Effective preventive strategies at personal and community level will definitely help to reduce these preventable causes of maternal mortalities. The key in decreasing these infectious diseases causes of maternal mortality includes early recognition and understanding of the clinical problems during the different phases of the disease and planning a rational approach to case management and a good clinical outcome. Thus an integrated and multi-disciplinary approach towards the case management can reduce these maternal mortalities, especially in tertiary care hospital. Early ANC registration, promotion of institutional deliveries, prompt diagnosis and treatment of the high risks associated with pregnancy. Operationalisation of sub-canters, primary health canters, community health canters and district hospitals for providing $24 \times 7$ basic and comprehensive obstetric cares.

Funding: No funding sources Conflict of interest: None declared

Ethical approval: The study was approved by the Institutional Ethics Committee

\section{REFERENCES}

1. Say L, Chou D, Gemmill A, Tunçalp Ö, Moller AB, Daniels J, et al. Global causes of maternal death: a WHO systematic analysis. Lancet Glob Health. 2014;2(6):e323-33.
2. Priddy KD. Immunologic adaptations during pregnancy. J Obstet Gynecol Neonatal Nurs. 1997;26:388.

3. Styrt B, Sugarman B. Estrogens and infection. Rev Infect Dis. 1991;13:1139-50.

4. World health organization, media centre. Fact sheet: The top 10 causes of death, 2016. Available at http://www.who.int/mediacentre/factsheets/fs310/en/ index1.html. Accessed 12 May 2016.

5. Press information bureau, government of India, ministry of health and family welfare. Achievements under millennium development goals, 2015. Available http://pib.nic.in/newsite/PrintRelease.aspx?relid=123 669. Accessed 11 May 2016.

6. Ahmed Y, Mwaba P, Chintu C, Grange JM, Ustianowski A, Zumla AA. study of maternal mortality at the University Teaching Hospital, Lusaka, Zambia: the emergence of tuberculosis as a major non-obstetric cause of maternal death. Int $\mathbf{J}$ Tuberculosis Lung Diseases. 1999;3(8):675-80.

7. Mathai M. Jaundice in pregnancy. In: Buckshy, Soonawala, Pathwardhan, eds. Principal and practice of obstetrics and gynaecology for postgraduates and practitioners (FOGSI Publication). New Delhi: Jaypeee Brothers, 1996.

8. Desai M, Ter Kuile FO, Nosten F, McGready R, Asamoa K, Brabin B, et al. Epidemiology and burden of malaria in pregnancy. Lancet Infect Dis. 2007;7(2):93-104.

9. Monif GRG, Baker DA, editors. Infectious Disease in Obstetrics and Gynecology. $6^{\text {th }}$ ed. New York: Parthenon; 2004:280-286.

10. World health organization, authors. Guidelines for the treatment of malaria. Geneva: World Health Organization; 2006.

11. Kiwuwa MS, Mufubenga P. Use of antenatal care, maternity services, intermittent presumptive treatment and insecticide treated bed nets by pregnant women in Luwero district, Uganda. Malar J. 2008;7:44.

12. Carroll ID, Toovey S, Van Gompel A. Dengue fever and pregnancy - a review and comment. Travel Med Infect Dis. 2007;5:183-8.

13. Chye JK, Lim CT, Ng KB, Lim JM, George R, Lam SK. Vertical transmission of dengue. Clin Infect Dis 1997;25:1374-7.

14. Fatimil LE, Mollah AH, Ahmed S, Rahman M. Vertical transmission of dengue: first case report from Bangladesh. Asian J Trop Med Public Health. 2003;34:800-3.

15. WHO dengue hemorrhagic fever: diagnosis, treatment, prevention and control. $2^{\text {nd }}$ edition. Geneva. $1997 . \quad$ Available at http://www.who.int/csr/resources/publications/dengu e/Deng uepublication/en/print.html Accessed 2 November 2009.

16. Zaba B, Calvert C, Marston M, Isingo R, NakiyingiMiiro J, Lutalo T, et al. Effect of HIV infection on pregnancy-related mortality in sub-Saharan Africa: 
Secondary analyses of pooled community based data from the network for Analysing Longitudinal Population-based HIV/AIDS data on Africa (ALPHA). The Lancet. 2013;381(9879):1763-71.

17. Calvert C, Ronsmans C. HIV and the risk of direct obstetric complications: A systemic review and meta-analysis. PLOS ONE. 2013;12(1):83.

18. Calvert C, Ronsmans C. The contribution of HIV to pregnancy-related mortality: A systemic review and meta-analysis. AIDS. 2013;27(10):1631-9.

19. Getahun H, Sculier D, Sismanidis C, Grzemska M, Raviglione M. Prevention, diagnosis, and treatment of tuberculosis in children and mothers: Evidence for action for maternal, neonatal, and child health services. Journal of Infectious Diseases. 2012;205:S216-27.

20. WHO Global Tuberculosis Report. Geneva: WHO 2013.

At http://www.who.int/tb/publications/global_report/en.

21. Gupta A, Bhosale R, Kinikar A, Gupte N, Bharadwaj R, Kagal A, et al. Maternal tuberculosis: a risk factor for mother to child transmission of Human
Immunodeficiency Virus. Journal of Infectious Diseases. 2013;205:S216-27.

22. Shaked Y, Shpilberg O, Samra D, Samra Y. Leptospirosis in pregnancy and its effect on the fetus: case report and review. Clin Infect Dis. 1993;17:2413.

23. Dawood FS, Jain S, Finelli L. Novel swine-origin influenza A (H1N1) virus investigation team, authors. Emergence of a novel swine-origin influenza A (H1N1) virus in humans. N Engl J Med. 2009;360:2605-15.

24. Moretti ME, Bar-Oz B, Fried S, Koren G. Maternal hyperthermia and the risk for neural tube defects in offspring: systematic review and meta-analysis. Epidemiology. 2005;16:216-9.

25. Centers for disease control and prevention web site, authors. Interim guidance on antiviral recommendations for patients with novel influenza $\mathrm{A}$ (H1N1) virus infection and their close contacts. Available at http://www.cdc.gov/h1n1flu/recommendations.htm. Accessed 24 August 2009.

Cite this article as: Satia MN, Panchbudhe SA, Shilotri MP. Maternal mortalities due to infectious diseases at a tertiary care centre in India. Int J Reprod Contracept Obstet Gynecol 2016;5:2395-401. 